\title{
Detection of carbapenemase production by blood culture carbapenem inactivation method (bcCIM) compared to modified carbapenem inactivation method (mCIM) in bloodstream infections
}

\author{
Safaa E. M. Metwally, Nehal A. Fahim, Nermeen M.A. Abdallah* \\ Medical Microbiology and Immunology, Faculty of Medicine, Ain Shams University, Cairo, Egypt
}

\section{Key words: \\ Carbapenemase, bloodstream infection, phenotypic test, Gram-negative bacilli \\ *Corresponding Author: Nermeen M.A. Abdallah Medical Microbiology and Immunology Department, Faculty of Medicine, Ain Shams University, Abbasia square, Cairo, Egypt Tel:+20223088175 \\ nermeen_mahmoud@med.asu.edu.eg}

\begin{abstract}
Background: Early detection of bloodstream infections (BSIs) caused by carbapenemase-producing organisms can guide rapid lifesaving appropriate therapy. A new variant of the modified Carbapenem Inactivation Method (mCIM) test, named blood culture CIM (bcCIM), can directly detect carbapenemase enzymes from positive blood culture. Objective: This study aimed at comparing detection of carbapenemase activity in Gram-negative bacilli (GNB) by Carbapenem Inactivation Method on positive blood culture and isolated colonies. Methodology: bcCIM test was used to detect carbapenemase production in 38 positive blood cultures. Subculture of blood samples followed by isolation and identification of causative pathogens was done, antimicrobial susceptibility by disk diffusion test as well as performing mCIM test were applied to all isolated GNB. Results: Forty-one GNB were isolated. Klebsiella spp. was the most common isolated GNB 16 (39\%). Most isolated GNB were carbapenemresistant 31(75.6\%). For monomicrobial bloodstream infection, there was fair (kappa $=0.327)$, moderate (kappa=0.429), and slight agreement (kappa=0.158) between results of $m C I M$ and results of bcCIM as regards all GNB, Enterobacteriaceae, and Pseudomonas, respectively. Conclusion: bcCIM is a promising test for direct detection of carbapenemase activity, particularly in carbapenem-resistant Enterobacteriaceae. Further studies are needed for the standardization of this method.
\end{abstract}

\section{INTRODUCTION}

Gram-negative bacilli (GNB), particularly Enterobacteriaceae, are the causative agents of a wide range of nosocomial as well as community-acquired infections that raise a significant public health concern ${ }^{\mathbf{1}}$.

The irrational wide use of antimicrobials, especially in developing countries, leads to the emergence of variety resistant GNB. The identification of these resistant phenotypes is mandatory for the proper implementation of infection control measures and better strategies for early patient management ${ }^{2}$.

Carbapenems are considered a cornerstone treatment for GNB infections, and they are used as empirical therapy for bloodstream infections, especially when suspected to be caused by extended-spectrum betalactamase (ESBL) producing Enterobacteriales. ${ }^{\mathbf{3 , 4}}$.

Several mechanisms are proposed for resistance to Carbapenems e.g., alteration of cell membrane porin channels, efflux pumps, and target-site mutation. Still, carbapenemase production is of utmost importance as they are usually carried on mobile elements having the risk of rapid transmission and emergence of outbreaks ${ }^{4}$.
Different assays were developed to shorten the time needed to identify causative pathogens and detect their resistance pattern directly from blood culture, e.g., SeptiFast, SepsiTest, and VYOO ${ }^{\mathbf{5}}$.

Carbapenem-resistant organisms increase morbidity and mortality rates in infected patients compared to carbapenem susceptible pathogens 6,7 and carbapenemase-producing GNB have the worst outcomes compared to non-carbapenemase producing GNB ${ }^{8}$. The delay in starting antimicrobial therapy in BSI patients increases the probability of devastating outcomes ${ }^{9}$.

In regions with higher levels of carbapenem resistance, early detection of the carbapenem resistance mechanism could be considered cost-effective in decreasing hospital stay and risk of mortality ${ }^{7}$.

In national surveillance, including 28 hospitals in Egypt, bloodstream infection was the most common healthcare-associated infection (HAIs) in 91 intensive care units (30\%). Klebsiella spp. was the most common isolated pathogen in all HAIs $(28.7 \%)$, with carbapenem resistance detected in $(48.1 \%)$ of them. In more comprehensive, recent surveillance, the percentage of carbapenem-resistant Klebsiella spp. was (53.7\%) with 
blood representing the most common specimen containing carbapenem-resistant Enterobacteriaceae $(\mathrm{CRE})^{10-12}$.

Several phenotypic and genotypic tests are available for direct detection of carbapenemase from positive blood culture, e.g., colorimetric methods such as $\beta$ CARBA ${ }^{\circledR}$ and NeoRapid CARB, which are limited by decreased specificity of the tests. Immunochromatographic test as NG-Test ${ }^{\circledR}$ CARBA 5. and molecular assays, e.g., Xpert@ Carba-R, are helpful, but they detect only the five common carbapenemases ${ }^{13-}$ 15 .

Meier \& Hamprecht ${ }^{\mathbf{1 3}}$ proposed a modification on the phenotypic test recommended by CLSI for carbapenemase detection; modified carbapenem inactivation method (mCIM) to be applied directly on positive blood cultures. The test is simple, cost-effective and does not require any equipment, and showed high sensitivity and specificity in detecting carbapenemase production in Enterobacteriaceae compared to three other phenotypic tests but compared them; the test carries the disadvantage of longer detection time.

In their publication, Meier \& Hamprecht ${ }^{13}$ used blood samples spiked with Enterobacteriaceae only. Yet, no other publications evaluated this phenotypic test directly on clinical samples and/or on GNB other than Enterobacteriaceae. Moreover, clinical samples carry the unlimited possibilities of mixed infection that is difficult to assess in spiked samples.

This study aimed at comparing the detection of carbapenemase activity in GNB by the Carbapenem Inactivation Method on positive blood culture and isolated colonies.

\section{METHODOLOGY}

This is a cross-sectional study conducted at Ain Shams University Hospitals during November and December 2020 on 300 positively cultured blood samples collected from patients suspected to have bloodstream infections. The Ethical Committee of Ain Shams University approved the study.

\section{Blood culture:}

Blood samples were cultured using DL-Bt Auto Blood Culture Detection System (Zhuhai DL Biotech, China). Gram-stained films of all positive cultures were examined, and blood culture bottles showing GNB detected by Gram stain were further processed.

Phenotypic detection of carbapenemase production directly from positive blood culture (bcCIM)

A $10-\mu \mathrm{g}$ meropenem disk (Oxoid, England) was immersed in $1 \mathrm{~mL}$ of the blood culture fluid containing ten $\mu \mathrm{L}$ of $10 \mathrm{mM} \mathrm{ZnSO}_{4}$ and incubated at $37^{\circ} \mathrm{C}$ for two $\mathrm{h}$.
A suspension equivalent to 0.5 McFarland of carbapenem susceptible E. coli reference strain ATCC $®$ 25922, was prepared and inoculated on a Muller Hinton agar plate (MHA) (Himedia, India), then left to dry for 3 minutes.

After incubation, the meropenem disk was pulled and placed on the inoculated MHA plate, then incubated at $37^{\circ} \mathrm{C}$ for $24 \mathrm{~h}$.

The sample was considered as carbapenemase positive if clear zone diameter measured 6-15 mm. Carbapenemase negative sample was considered if clear zone diameter was $\geq 19 \mathrm{~mm}$. The result was recorded as indeterminate if zone diameter $\geq 19 \mathrm{~mm}$ with pinpoint colonies or 16-18 $\mathrm{mm}$ clear zone (considered positive if pinpoint colonies present) ${ }^{\mathbf{1 3}}$.

\section{Isolation and identification of GNB}

Subculture from positive blood cultures on blood and MacConkey's agar plates (Oxoid, England) was done. Isolated colonies were identified through conventional microbiological methods, i.e., Gram stain and suitable biochemical reactions ${ }^{\mathbf{1 6}}$.

\section{Detection of antimicrobial susceptibility}

Detection of antimicrobials susceptibility of isolates by disk diffusion method on MHA was done and interpreted according to CLSI guidelines ${ }^{17}$. Table (1) shows the used antimicrobial disks for different GNB (Oxoid, England).

Table 1: Antibiotic disks used for different GNB

\begin{tabular}{|l|c|}
\hline \multicolumn{1}{|c|}{ Antibiotic } & Disk content \\
\hline Amoxicillin $^{\mathbf{a}}$ & $10 \mu \mathrm{g}$ \\
\hline Amoxicillin/Clavulanic Acid $^{\mathbf{a}}$ & $20 / 10 \mu \mathrm{g}$ \\
\hline Piperacillin/Tazobactam $^{\mathbf{a}, \mathbf{b}, \mathbf{c}}$ & $100 / 10 \mu \mathrm{g}$ \\
\hline Ceftriaxone $^{\mathbf{a , c}}$ & $30 \mu \mathrm{g}$ \\
\hline Ceftazidime $^{\mathbf{a}, \mathbf{b}, \mathbf{c}}$ & $30 \mu \mathrm{g}$ \\
\hline Ampicillin/Sulbactam $^{\mathbf{c}}$ & $10 / 10 \mu \mathrm{g}$ \\
\hline Cefepime $^{\mathbf{a}, \mathbf{b}, \mathbf{c}}$ & $30 \mu \mathrm{g}$ \\
\hline Meropenem $^{\mathbf{a}, \mathbf{b}, \mathbf{c}}$ & $10 \mu \mathrm{g}$ \\
\hline Amikacin $^{\mathbf{a}, \mathbf{b}, \mathbf{c}}$ & $30 \mu \mathrm{g}$ \\
\hline Gentamicin $^{\mathbf{a}, \mathbf{b}, \mathbf{c}}$ & $10 \mu \mathrm{g}$ \\
\hline Sulfamethoxazole/Trimethoprim $^{\mathbf{a}, \mathbf{c}}$ & $25 \mu \mathrm{g}$ \\
\hline Ciprofloxacin $^{\mathbf{a}, \mathbf{b}, \mathbf{c}}$ & $5 \mu \mathrm{g}$ \\
\hline Doxycycline $^{\mathbf{c}}$ & $30 \mu \mathrm{g}$ \\
\hline
\end{tabular}

a: used for Enterobacteriaceae

${ }^{\mathrm{b}}$ : used for Pseudomonas spp.

c: used for Acinetobacter spp. 
Phenotypic detection of carbapenemase production in GNB isolates using ( $\mathrm{mCIM}$ ) method

The test was performed for all isolated strains and interpreted according to CLSI guidelines ${ }^{17}$

Briefly, for each isolate, a 1- $\mu \mathrm{L}$ loopful of bacteria for Enterobacteriaceae and $10 \mu \mathrm{L}$ for P. aeruginosa and A. baumannii, was emulsified in $2 \mathrm{~mL}$ Tryptone Soya broth (TSB) (Himedia, India), then vortexed for $10-15$ seconds.

Meropenem disk $(10 \mu \mathrm{g})$ was added to each tube and processed and interpreted as for bcCIM. Additionally, the mCIM test was repeated for strains reported to be indeterminate for carbapenemase production $\mathbf{1 8}$

Carbapenemase positive and negative strains were used as control while performing mCIM and bcCIM tests.

\section{Statistical analysis}

Data were analyzed using SPSS (statistical package for social science) version 25 (Armonk, NY: IBM Corp) software for Windows. Means and standard deviations were calculated for continuous variables. Percentages were used for categorical variables.

The Chi-square test was used to study the comparison and association between two qualitative variables. A P-value of $<0.05$ was considered statistically significant.

Agreement between mCIM and bcCIM were tested with kappa statistics; cut-off values for the kappa was interpreted as values $\leq 0$ indicating no agreement and
$0.01-0.20$ as slight, $0.21-0.40$ as fair, $0.41-0.60$ as moderate, $0.61-0.80$ as substantial, and $0.81-1.00$ as almost perfect agreement. ${ }^{19}$

\section{RESULTS}

Out of 300 positively signaled blood culture bottles, 38 samples were positive for GNB (Fig1). The age of the 38 patients ranged from 24 to 73 years with a mean \pm SD of $49.92 \pm 15.13$; they were 17 females $(44.7 \%)$ and 21 males $(55.3 \%)$.

Forty-one GNB were isolated from positive blood culture bottles. Isolates were distributed as follows: Klebsiella spp.16 (39\%), Pseudomonas spp. 9(22\%), E.coli 8(19.5\%), Acinetobacter 7 (17.1\%) and Proteus spp. 1(2.4\%).

Mixed infection was detected in 3 samples (two samples contained E. coli and Klebsiella spp. while one sample contained Pseudomonas and Proteus spp.

For all GNB highest resistance was recorded for Ceftazidime, while the least resistance was against amikacin (Fig2).

Most of the isolated GNB were carbapenemresistant 31(75.6\%), all Acinetobacter isolates were carbapenem-resistant $7(100 \%)$, while $7(77.8 \%)$ and $17(68.0 \%)$ of Pseudomonas spp. and Enterobacteriaceae isolates were carbapenem-resistant, respectively.

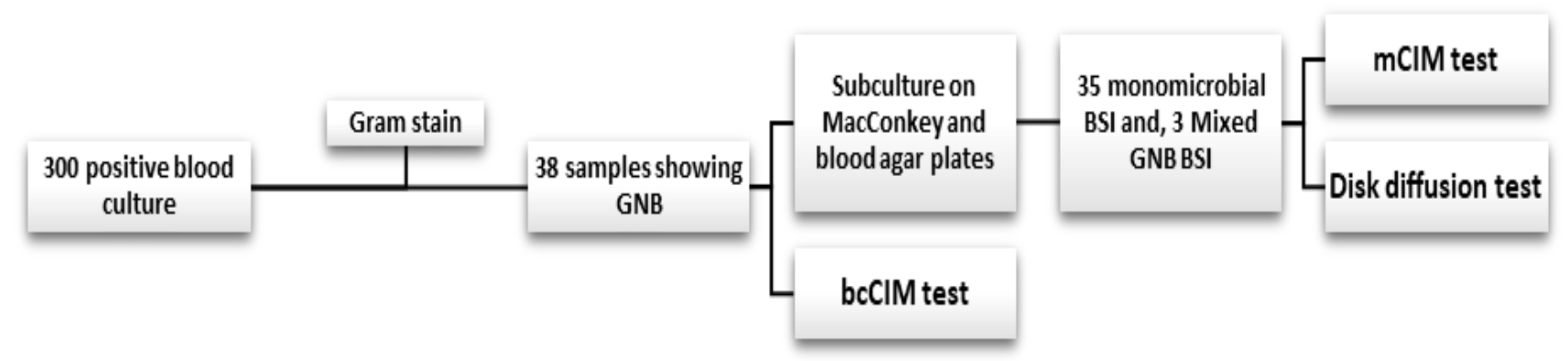

Fig. 1: Flow chart for samples processing 


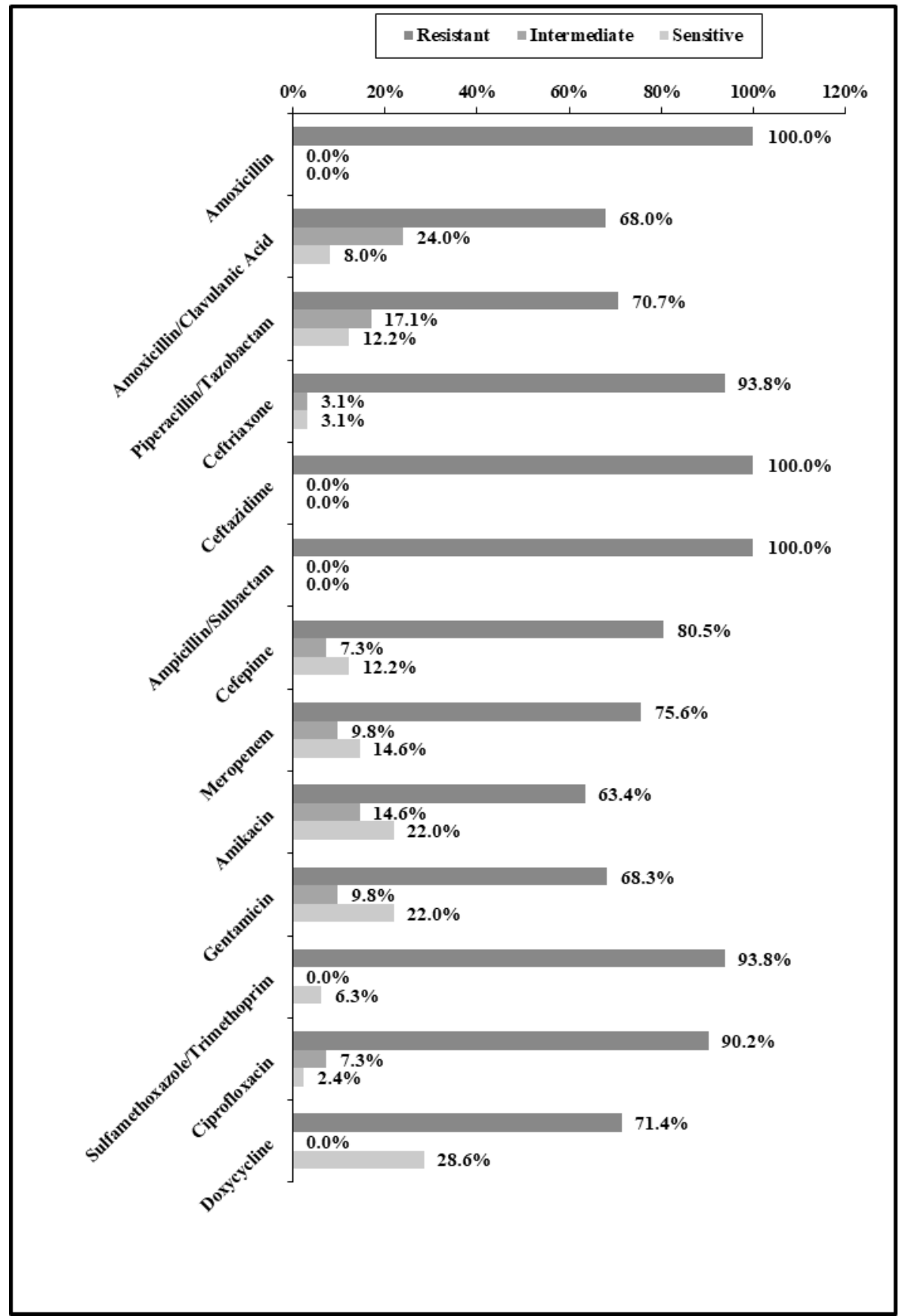

Fig. 2: The susceptibility pattern of isolates to different antimicrobials

For the 41 isolates tested by mCIM; $31(75.6 \%)$ of GNB were positive for carbapenemase production while $6(14.6 \%), 4(9.8 \%)$, were negative, indeterminate for carbapenemase production, respectively. For the 38 blood samples tested using bcCIM, half of the samples, $19(50 \%)$, were negative, and nine samples (23.7\%) tested positive for carbapenemase production, while ten samples $(26.3 \%)$ gave indeterminate results

Table (2) demonstrates results of carbapenem susceptibility, mCIM, and bcCIM in different isolated GNB.
When comparing bcCIM and mCIM, samples with mixed GNB infection (3 samples) were excluded. Indeterminate results were considered as positive results.

There was a fair agreement between mCIM and bcCIM for GNB; a moderate agreement was noticed for Enterobacteriaceae while for Pseudomonas spp. there was a slight agreement between both tests (table 3 ). 
Table 2: Results of carbapenem susceptibility, mCIM, and bcCIM in different GNB

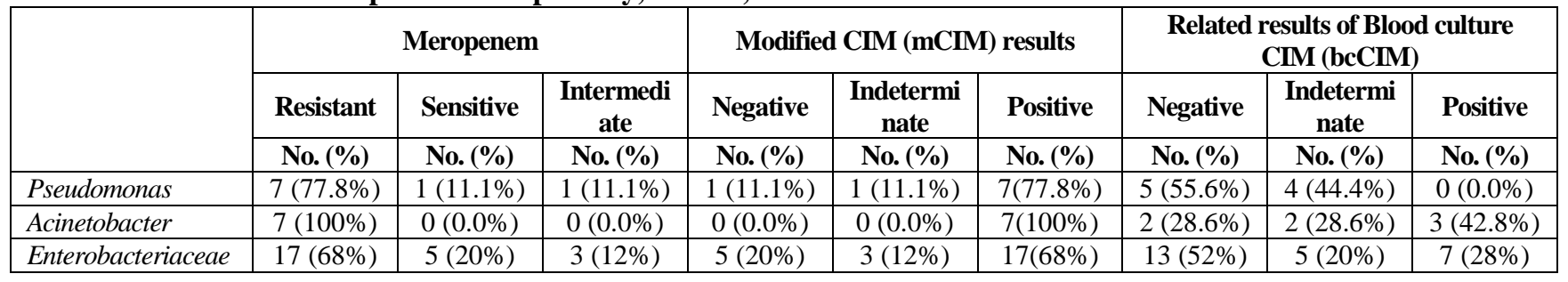

Table 3: Correlation between results of mCIM and bcCIM

\begin{tabular}{|c|c|c|c|c|c|c|}
\hline \multirow{2}{*}{\multicolumn{2}{|c|}{ mCIM }} & \multicolumn{2}{|c|}{ bcCIM } & \multirow{3}{*}{$\begin{array}{c}\text { Total } \\
6\end{array}$} & \multirow{2}{*}{$P$-value } & \multirow{2}{*}{$\begin{array}{l}\text { The measure of } \\
\text { Agreement Kappa }\end{array}$} \\
\hline & & \multirow{2}{*}{$\frac{\text { Negative }}{6}$} & \multirow{2}{*}{$\frac{\text { Positive* }^{*}}{0}$} & & & \\
\hline \multirow[t]{2}{*}{ GNB } & Negative & & & & \multirow[t]{3}{*}{0.009} & \multirow[t]{3}{*}{0.327} \\
\hline & Positive* & 12 & 17 & 29 & & \\
\hline \multicolumn{2}{|l|}{ Total } & 18 & 17 & 35 & & \\
\hline \multirow[t]{2}{*}{ Enterobacteriaceae } & Negative & 5 & 0 & 5 & \multirow[t]{3}{*}{0.020} & \multirow[t]{3}{*}{0.429} \\
\hline & Positive* & 6 & 9 & 15 & & \\
\hline \multicolumn{2}{|l|}{ Total } & 11 & 9 & 20 & & \\
\hline \multirow[t]{2}{*}{ Pseudomonas } & Negative & 1 & 0 & 1 & \multirow[t]{3}{*}{0.408} & \multirow[t]{3}{*}{0.158} \\
\hline & Positive* & 4 & 3 & 7 & & \\
\hline \multicolumn{2}{|l|}{ Total } & 5 & 3 & 8 & & \\
\hline \multirow[t]{2}{*}{ Acinetobacter } & Negative & 0 & 0 & 0 & \multirow[t]{3}{*}{ NA } & \multirow[t]{3}{*}{ NA } \\
\hline & Positive* & 2 & 5 & 7 & & \\
\hline \multicolumn{2}{|l|}{ Total } & 2 & 5 & 7 & & \\
\hline
\end{tabular}

*Indeterminate results were considered as positive ${ }^{20}$, NA: Not applicable

\section{DISCUSSION}

Bloodstream infection is responsible for significant short and long-term morbidity and mortality worldwide 21. Higher rates of resistance to antimicrobials are documented in GNB causing BSIs, especially towards carbapenem antimicrobials ${ }^{\mathbf{1 1}, \mathbf{2 2}}$.

In this study, GNB constituted (13.9\%) of all documented BSIs. In 38 blood samples, Klebsiella spp. (39\%) was the predominant pathogen followed by Pseudomonas spp. (21.9\%). Similar results were reported by other studies in Egypt and nearby countries as Klebsiella spp. represented the most common GNB in BSIs ${ }^{23,24}$.

Isolated GNB showed high resistance to most antibiotics. The least resistance detected was for amikacin antibiotic. Similar results were reported by other studies on GNB BSIs in Egypt ${ }^{23,25}$. Amikacin antibiotic demonstrate good activity against GNB because it is less susceptible to inactivating enzyme due to its chemical structure ${ }^{\mathbf{2 6}}$.

In the present study, most of the isolated GNB (75.6\%) were carbapenem-resistant, and all Acinetobacter spp. were carbapenem-resistant. Similar results were obtained by Tohamy and his colleagues who studied BSIs in cancer patients ${ }^{27}$.Carbapenem resistance in Enterobacteriaceae $(68.0 \%)$ and
Pseudomonas spp. (77.8\%) where higher than detected elsewhere. ${ }^{11,25}$ but in a more recent study conducted at ICUs of a tertiary care hospital in Egypt, similar high rates for carbapenem resistance were detected ${ }^{\mathbf{2 8}}$.

Bloodstream infections by carbapenemaseproducing GNB show variable rates in different world regions ${ }^{13,29-31}$. According to the results of mCIM, 31 (75.6\%) of GNB were positive for carbapenemase production. This finding is higher than reported in a similar study in Egypt by Abdulall and his colleagues, but they used different phenotypic tests for carbapenemase detection ${ }^{\mathbf{2 5}}$.

All strains that were resistant to carbapenem were carbapenemase producers. This finding is higher than reported in studies by Kamel and later on by Raheel and their colleagues. They found that (67.9\%) (46.6\%) respectively of the CRE isolates from different HAIs were positive for carbapenemase production by the mCIM test ${ }^{32,33}$.

In nationwide surveillance in Italy $(65.1 \%)$ of CREcausing BSIs were confirmed carbapenemase producer phenotypically $^{34}$. At the same time, in the PANORAMA study, carbapenemase-encoding genes were detected in (88\%) of CRE-causing BSIs in 10 low-income and middle-income countries, including Egypt ${ }^{35}$. Carbapenem-resistant organisms are difficult to treat than carbapenem-sensitive ones, and carbapenemase- 
producing GNB has the worst outcomes compared to non-carbapenemase-producing GNB ${ }^{8}$.

Several variations exist of the phenotypic assay Carbapenem Inactivation Method (CIM), i.e., modified CIM (mCIM), rapid CIM (rCIM), Simplified CIM (sCIM), (CIMTris), and (CIMTrisII) for detection of carbapenemase-producing $\mathrm{GNB}^{17,20,36,37}$. Moreover, sodium mercapto acetate (SMA-mCIM), EDTA (eCIM) and CIMplus tests are modified versions of the test designed to discriminate different types of carbapenemases ${ }^{\mathbf{1 7}, 38}$. A recent variation of this test (bcCIM) was applied directly on positive blood culture aiming at early detection of carbapenemase activity before culture results appear ${ }^{\mathbf{1 3}}$.

Different CIM tests share the main principle with slightly different procedures. These variations in steps and materials used lead to variable sensitivity and specificity towards detection of carbapenemases in GNB culture. for instance, the difference between mCIM and CIM was using tryptone soy broth instead of water and different inoculum and incubation time ${ }^{\mathbf{2 0}}$.

In the present study, there was a fair agreement (kappa $=0.327$ ) between mCIM as a reference method recommended by CLSI and bcCIM in detecting carbapenemase-producing GNB. The decreased performance of bcCIM compared to $\mathrm{mCIM}$, and to the results of Meier \& Hamprecht could be due to several factors, e.g., using different blood culture system, besides clinical samples differ from spiked samples in terms of inoculum used and other host factors ${ }^{\mathbf{1 3}}$.

Similar results were reported by De Lima-Morales et al. ${ }^{39}$ comparing another carbapenemase detecting phenotypic test directly on the blood and on pure culture, where applying the test directly on blood detected only $80 \%$ of carbapenemase positive isolates identified by the same test on pure culture.

In the present study, results of mCIM correlated better with bcCIM as regard Enterobacteriaceae (moderate agreement, kappa $=0.429$ ) compared to Pseudomonas spp. (slight agreement, kappa=0.158).

Previous studies recommended when performing the mCIM test to use a higher inoculum (10ul) for reliable detection of carbapenemase production by $P$. aeruginosa and $A$. baumannii than the inoculum used for the mCIM test in Enterobacteriaceae (1ul) ${ }^{\mathbf{1 7}, 18}$.

In blood samples, the causative agent couldn't be identified before performing bcCIM, and worth noting that blood culture bottles that signaled positive might not be processed immediately due to the limitation of working hours, i.e., not processed until next working hours. This may affect the initial test inoculum and, subsequently, the results. This study is the first to test carbapenemase production using bcCIM on blood samples containing Pseudomonas and Acinetobacter spp. Future studies are needed to determine the optimum processing time for best results for different GNB.
Acinetobacter baumannii is an important cause of BSI. The mCIM test is not standardized for testing carbapenemase production among Acinetobacter spp. as the most common types of carbapenemases produced by Acinetobacter have weak carbapenemase activity ${ }^{18}$.

In the present study, all Acinetobacter isolates tested positive for carbapenemase production using mCIM. The results of the bcCIM test matched the results of mCIM test in $5(71.4 \%)$ samples. Unfortunately, the statistical correlation between the two tests couldn't be performed as none of the Acinetobacter isolates was negative for carbapenemase production using the MCIM test.

The present study included three samples with mixed GNB infection. Results of bcCIM in two samples correlated with results of mCIM. The third sample tested negative for bcCIM while both strains were positive by mCIM. Different combinations of various types of carbapenemases may affect reporting of results, and future large-scale studies on mixed GNB bacteremia are needed to revise the cut-off points of reporting bcCIM in these situations.

Although simplicity and feasibility of performing mCIM and bcCIM tests, both detect only carbapenemases production, which is one of the mechanisms responsible for carbapenem resistance in GNB, besides no differentiation between different types of carbapenemases can be achieved using mCIM, bcCIM tests alone ${ }^{\mathbf{8 , 1 3}}$.

Limitation of the current study includes the small number of isolates tested and the lack of molecular characterization of the types of carbapenemases present in isolated strains.

\section{CONCLUSION}

The relatively high incidence of carbapenemresistant GNB causing BSI warrants the need for effective antimicrobial stewardship as well as effective implementation of an infection control program.

Delay in starting treatment may lead to catastrophic consequences. Phenotypic tests for carbapenemase production give a good alternative to genotypic methods that are sometimes nonapplicable, especially in lowresource countries. The bcCIM test could be a promising test for the early detection of carbapenemase production, particularly in CRE. Further studies are needed for the optimization of culture conditions and cut-off points for reporting results.

- The authors declare that they have no financial or non-financial conflicts of interest related to the work done in the manuscript.

- Each author listed in the manuscript has seen and approved the submission of this version of the manuscript and takes full responsibility for it. 
- This article has not been published anywhere and is not currently under consideration by another journal or a publisher.

\section{REFERENCES}

1. Nordmann P, Naas T, Poirel L. Global spread of carbapenemase producing Enterobacteriaceae. Emerg Infect Dis. 2011;17(10):1791-1798. doi:10.3201/eid1710.110655

2. Davoudi-Monfared E, Khalili H. The threat of carbapenem-resistant gram-negative bacteria in a Middle East region. Infect Drug Resist. 2018;Volume 11:1831-1880. doi:10.2147/IDR.S176049

3. Strich JR, Heil EL, Masur H. Considerations for Empiric Antimicrobial Therapy in Sepsis and Septic Shock in an Era of Antimicrobial Resistance. J Infect Dis. 2020;222(Supplement_2):S119-S131. doi:10.1093/infdis/jiaa221

4. Codjoe F, Donkor E. Carbapenem Resistance: A Review. Med Sci. 2017;6(1):1. doi:10.3390/medsci6010001

5. Opota O, Jaton K, Greub G. Microbial diagnosis of bloodstream infection: Towards molecular diagnosis directly from blood. Clin Microbiol Infect. doi:10.1016/j.cmi.2015.02.005

6. Cai B, Echols R, Magee G, et al. Prevalence of Carbapenem-Resistant Gram-Negative Infections in the United States Predominated by Acinetobacter baumannii and Pseudomonas aeruginosa. Open Forum Infect Dis. 2017;4(3). doi:10.1093/ofid/ofx176

7. Nordmann P, Poirel L. Epidemiology and Diagnostics of Carbapenem Resistance in Gramnegative Bacteria. Clin Infect Dis. 2019;69(Supplement_7):S521-S528. doi:10.1093/cid/ciz824

8. Sfeir MM, Hayden JA, Fauntleroy KA, et al. EDTA-modified carbapenem inactivation method: A phenotypic method for detecting metallo- $\beta$ lactamase-producing enterobacteriaceae. J Clin Microbiol. 2019;57(5). doi:10.1128/JCM.01757-18

9. Schwarzenbacher J, Kuhn S-O, Vollmer M, et al. On-site blood culture incubation shortens the time to knowledge of positivity and microbiological results in septic patients. Calderaro A, ed. PLoS One. 2019;14(12):e0225999. doi:10.1371/journal.pone.0225999

10. Talaat M, El-Shokry M, El-Kholy J, et al. National surveillance of health care-associated infections in Egypt: Developing a sustainable program in a resource-limited country. Am J Infect Control. 2016;44(11):1296-1301. doi:10.1016/j.ajic.2016.04.212

11. Kotb S, Lyman M, Ismail G, et al. Epidemiology of Carbapenem-resistant Enterobacteriaceae in
Egyptian intensive care units using National Healthcare-associated Infections Surveillance Data, 2011-2017. Antimicrob Resist Infect Control. 2020;9(1):2. doi:10.1186/s13756-019-0639-7

12. Kotb MM, Mowafy HL. Detection of Carbapenemase Producing Enterobacteriaceae using the Modified Carbapenem Inactivation Method. Egypt J Med Microbiol. 2019;28(4):171177.

13. Meier M, Hamprecht A. Systematic comparison of four methods for detection of carbapenemaseproducing enterobacterales directly from blood cultures. J Clin Microbiol. 2019;57(11). doi:10.1128/JCM.00709-19

14. Stokes W, Pitout J, Campbell L, Church D, Gregson D. Rapid detection of carbapenemaseproducing organisms directly from blood cultures positive for Gram-negative bacilli. Eur $\mathrm{J}$ Clin Microbiol Infect Dis. 2020;40(2):381-384. doi:10.1007/s10096-020-04005-4

15. Cointe A, Walewski V, Hobson CA, et al. Rapid carbapenemase detection with xpert carba-R V2 directly on positive blood vials. Infect Drug Resist. 2019;12:3311-3316. doi:10.2147/IDR.S204436

16. Tille PM. Traditional cultivation and Identification. In: Bailey and Scott's Diagnostic Microbiology. Fourteenth. Elsevier Inc.; 2017:86-112.

17. CLSI. Performance Standards for Antimicrobial Susceptibility Testing. In: CLSI Supplement M100. 30th ed. Clinical and Laboratory Standards Institute; 2020

18. Simner PJ, Kristie Johnson J, Brasso WB, et al. Multicenter evaluation of the modified carbapenem inactivation method and the carba NP for detection of carbapenemase-producing pseudomonas aeruginosa and acinetobacter baumannii. J Clin Microbiol. 2018;56(1):1369-1386. doi:10.1128/JCM.01369-17

19. Landis JR, Koch GG. The Measurement of Observer Agreement for Categorical Data. Biometrics. 1977;33(1):159. doi:10.2307/2529310

20. Pierce VM, Simner PJ, Lonsway DR, et al. Modified carbapenem inactivation method for phenotypic detection of carbapenemase production among enterobacteriaceae. J Clin Microbiol. 2017;55(8):2321-2333. doi:10.1128/JCM.00193-17

21. Fleischmann C, Scherag A, Adhikari NKJ, et al. Assessment of global incidence and mortality of hospital-treated sepsis current estimates and limitations. Am J Respir Crit Care Med. 2016;193(3):259-272. doi:10.1164/rccm.2015040781OC

22. Alagesan M, Gopalakrishnan R, Panchatcharam SN, Dorairajan S, Ananth TM, Venkatasubramanian R. A decade of change in 
susceptibility patterns of Gram-negative blood culture isolates: A single center study. GERMS. 2015;5(3):65-77. doi:10.11599/germs.2015.1073

23. Ahmed SH, Daef EA, Badary MS, Mahmoud MA, Abd-Elsayed AA. Nosocomial blood stream infection in intensive care units at Assiut University Hospitals (Upper Egypt) with special reference to extended spectrum $\beta$-lactamase producing organisms. BMC Res Notes. 2009;2(1):76. doi:10.1186/1756-0500-2-76

24. Bandy A, Almaeen AH. Pathogenic spectrum of blood stream infections and resistance pattern in Gram-negative bacteria from Aljouf region of Saudi Arabia. Woźniakowski G, ed. PLoS One. 2020;15(6):e0233704. doi:10.1371/journal.pone.0233704

25. Abdulall AK, Tawfick MM, El Manakhly AR, El Kholy A. Carbapenem-resistant Gram-negative bacteria associated with catheter-related bloodstream infections in three intensive care units in Egypt. Eur $\mathrm{J}$ Clin Microbiol Infect Dis. 2018;37(9):1647-1652. doi:10.1007/s10096-0183294-7

26. Ramirez MS, Tolmasky ME. Amikacin: Uses, resistance, and prospects for inhibition. Molecules. 2017;22(12). doi:10.3390/molecules22122267

27. Tohamy ST, Aboshanab KM, El-Mahallawy A, ElAnsary MR, Selim Afifi S. Infection and Drug Resistance Dovepress Prevalence of multidrugresistant gram-negative pathogens isolated from febrile neutropenic cancer patients with bloodstream infections in egypt and new synergistic antibiotic combinations. Infect Drug Resist. Published online 2018:11-791. doi:10.2147/IDR.S163293

28. Negm EM, Mowafy SMS, Mohammed AA, et al. Antibiograms of intensive care units at an Egyptian tertiary care hospital. Egypt J Bronchol. 2021;15(1):15. doi:10.1186/s43168-021-00059-w

29. Brink AJ. Epidemiology of carbapenem-resistant Gram-negative infections globally. Curr Opin Infect Dis. Published online 2019. doi:10.1097/QCO.0000000000000608

30. Lin Y-T, Su C-F, Chuang C, et al. Appropriate Treatment for Bloodstream Infections Due to Carbapenem-Resistant Klebsiella pneumoniae and Escherichia coli: A Nationwide Multicenter Study in Taiwan. Open Forum Infect Dis. 2019;6(2). doi:10.1093/ofid/ofy336

31. Manenzhe RI, Zar HJ, Nicol MP, Kaba M. The spread of carbapenemase-producing bacteria in
Africa: A systematic review. J Antimicrob Chemother. 2015;70(1):23-40. doi:10.1093/jac/dku356

32. Raheel A, Azab H, Hessam W, Abbadi S, Ezzat A. Detection of carbapenemase enzymes and genes among carbapenem-resistant Enterobacteriaceae isolates in Suez Canal University Hospitals in Ismailia ,Egypt. Microbes Infect Dis. 2020;1(1):2433. doi:10.21608/mid.2020.25702.1007

33. Kamel NA, El-tayeb WN, El-Ansary MR, Mansour MT, Aboshanab KM. Phenotypic screening and molecular characterization of carbapenemaseproducing Gram-negative bacilli recovered from febrile neutropenic pediatric cancer patients in Egypt. Abdel-Naim AB, ed. PLoS One. 2018;13(8):e0202119. doi:10.1371/journal.pone.0202119

34. Iacchini S, Sabbatucci M, Gagliotti $C$, et al. Bloodstream infections due to carbapenemaseproducing Enterobacteriaceae in Italy: Results from nationwide surveillance, 2014 to 2017. Eurosurveillance. 2019;24(5). doi:10.2807/1560-7917.ES.2019.24.5.1800159

35. Stewardson AJ, Marimuthu K, Sengupta S, et al. Effect of carbapenem resistance on outcomes of bloodstream infection caused by Enterobacteriaceae in low-income and middle-income countries (PANORAMA): a multinational prospective cohort study. Lancet Infect Dis. 2019;19(6):601-610. doi:10.1016/S1473-3099(18)30792-8

36. Muntean MM, Muntean AA, Gauthier L, et al. Evaluation of the rapid carbapenem inactivation method (rCIM): A phenotypic screening test for carbapenemase-producing Enterobacteriaceae. J Antimicrob Chemother. 2018;73(4):900-908. doi:10.1093/jac/dkx519

37. Uechi K, Tada T, Kuwahara-Arai K, et al. An improved carbapenem inactivation method, CIMTrisII, for carbapenemase production by gramnegative pathogens. J Med Microbiol. 2019;68(2):124-131. doi:10.1099/jmm.0.000888

38. Caméléna F, Cointe A, Mathy V, et al. Within-aDay Detection and Rapid Characterization of Carbapenemase by Use of a New Carbapenem Inactivation Method-Based Test, CIMplus Downloaded from. jcm.asm.org $1 \mathrm{~J}$ Clin Microbiol. 2018;56:137-155. doi:10.1128/JCM.00137-18

39. De Lima-Morales D, Ávila H, Soldi T, et al. Rapid detection of carbapenemase production directly from blood culture by colorimetric methods: Evaluation in a routine microbiology laboratory. J Clin Microbiol. 2018;56(9). doi:10.1128/JCM.00325-18 